UNIVERSUM • Vol. $30 \bullet$ No $1 \bullet 2015 \bullet$ Universidad de Talca

José Ramón Saavedra y Adolfo Valderrama: Lengua y educación en el Chile del siglo XIX

Tamara Bustos, Carlos Valladares y Darío Rojas

Pp. 39 a 53

\title{
JOSÉ RAMÓN SAAVEDRA Y ADOLFO VALDERRAMA: LENGUA Y EDUCACIÓN EN EL CHILE DEL SIGLO XIX ${ }^{1}$
}

\author{
José Ramón Saavedra and Adolfo Valderrama: \\ Language and education in 19th century Chile
}

\author{
Tamara Bustos* \\ Juan Carlos Valladares** \\ Darío Rojas***
}

\section{RESUMEN}

En el presente estudio, analizamos las ideas sobre la relación entre lenguaje y educación en dos autores hasta ahora poco atendidos en la historiografía lingüística chilena: José Ramón Saavedra (1821-1907) y Adolfo Valderrama (1834-1902). Mostramos que para estos autores la educación lingüística era relevante por su condición instrumental en la consecución del orden social, aspiración tanto de los tradicionalistas como de los

\footnotetext{
${ }^{1}$ El presente trabajo forma parte del proyecto "Ideologías lingüísticas sobre el español entre los actores sociales de la asignatura de Lenguaje y Comunicación en Chile: aproximación a su impacto en las prácticas y resultados en la sala de clases. El aporte de la Lingüística antropológica al análisis del currículo oculto en la investigación educativa", financiado por el Fondo de Innovación en Investigación Disciplinaria de la Iniciativa Bicentenario Juan Gómez Millas, Universidad de Chile.

* Licenciada en Lengua y Literatura Hispánica con mención en Lingüística, Universidad de Chile. Correo electrónico: tamara.bustos@ug.uchile.cl

** Licenciado en Lengua y Literatura Hispánica con mención en Lingüística, Universidad de Chile. Correo electrónico: juan.valladares@ug.uchile.cl

*** Departamento de Lingüística, Facultad de Filosofía y Humanidades, Universidad de Chile. Santiago, Chile. Correo electrónico: darioroj@u.uchile.cl
}

Artículo recibido el 1 de marzo de 2014. Aceptado el 19 de noviembre de 2014. 
innovadores. De ahí que sus propias ideologías y actitudes lingüísticas variarán a partir de sus propias ideas del orden (y de la libertad).

Palabras clave: Historiografía lingüística, enseñanza de la lengua, José Ramón Saavedra, Adolfo Valderrama.

\begin{abstract}
In the present piece of research we analyze the ideas about language and education held by José Ramón Saavedra (1821-1907) and Adolfo Valderrama (1834-1902), two authors that have been usually overlooked by Chilean linguistic historiography. We show that for these authors language education was relevant because it was useful for achieving social order, an ideal seeked by both conservatives and non-conservatives. This explains how their linguistic attitudes and ideologies differ from the starting point of their ideas of order and freedom.
\end{abstract}

Keywords: Linguistic historiography, language teaching, José Ramón Saavedra, Adolfo Valderrama.

\title{
INTRODUCCIÓN
}

El siglo XIX chileno fue un momento de proliferación para lo que Blommaert (1999) llama debates lingüístico-ideológicos: controversias públicas en que el lenguaje es tema de discusión y en que, por supuesto, el lenguaje es más bien una excusa para debatir acerca de cuestiones de orden político, moral, epistémico, etc. Como señala Metzeltin (2011), el lenguaje fue uno de los varios frentes discursivos en torno a los que se forjó la nueva nación chilena independiente, entendida esta como una comunidad imaginada (Anderson, 1993). Andrés Bello, Domingo F. Sarmiento y muchos otros personajes ilustres del momento tomaron parte en dicha invención metadiscursiva, y los frentes propiamente lingüísticos en que se dio la contienda en esta nueva nación fueron diversos: ortografía, gramática, vocabulario, etc. Sin embargo, estos frentes con frecuencia confluyeron hacia un ámbito de interés transversal: la educación lingüística, lo cual se explica por la centralidad que la educación en general tenía en el proyecto político de los nuevos gobernantes. En consecuencia, la relación entre lenguaje y educación, o, mejor dicho, el carácter instrumental del lenguaje en la educación de los ciudadanos, fue uno de los problemas centrales de los debates lingüístico-ideológicos antes seńalados.

En el presente estudio nos proponemos analizar las ideas acerca de la relación entre lenguaje y educación sostenidas por dos autores hasta ahora poco atendidos: José Ramón Saavedra (1821-1907) y Adolfo Valderrama (1834-1902). Ambos nos 
parecen interesantes no solo por representar un periodo relativamente desatendido en la historiografía lingüística chilena del XIX, como es la segunda mitad de dicho siglo, sino también por la estrecha vinculación que existe entre ambos autores y el contexto que les precede, marcado por la influencia de los debates en torno al rol de la lengua en la educación y la formación de los ciudadanos de la nación chilena.

Nuestra investigación tiene por marco la historiografía lingüística (también llamada historia de las ideas lingüisticas o historia de la lingüistica), que tiene por objeto dar cuenta científicamente de cómo el conocimiento acerca del lenguaje ha sido obtenido y cómo este conocimiento ha cambiado o se ha mantenido a lo largo de la historia (Swiggers, 2009), sin desconocer la naturaleza política, social y culturalmente situada de la formación de los discursos de verdad acerca del lenguaje. Esta área de investigación ha cobrado importancia en las últimas décadas debido a que, para los propios estudios lingüísticos y filológicos, es central informarse y reflexionar sobre su actividad histórica, así como entenderla en el marco de las circunstancias socioculturales en que tuvo desarrollo, con el fin de comprender apropiadamente su situación actual y su proyección hacia el futuro. La relevancia de estos estudios, sin embargo, excede por mucho el ámbito de la lingüística, pues la historia de las ideas acerca del lenguaje se entremezcla con la historia cultural e intelectual en sentido amplio, en la medida en que dichas ideas forman parte y se relacionan con imaginarios más amplios que revelan aspectos epistemológicos, morales, políticos y culturales, en general, de las comunidades en que han surgido. En consecuencia, la historiografía lingüística chilena forma parte de la historia intelectual de Chile. Nuestra investigación se propone hacer una pequeña contribución a tan necesario proyecto.

\section{CONTEXTO LINGÜÍSTICO-IDEOLÓGICO DEL S. XIX CHILENO}

Tras la Independencia, entre los principales desafíos que enfrentaron los nuevos administradores del territorio chileno se encontraban la formación y consolidación de un Estado autónomo, la adopción del republicanismo como sistema de gobierno, y la construcción de la nación como una nueva categoría identitaria (Cid, 2012). En este contexto histórico y político, entonces, debe entenderse la actividad metalingüística del siglo XIX chileno. Huisa ha denominado impronta política a la forma en que este discurso hispanoamericano sobre la lengua (el lexicográfico, señala el autor en particular) "manifiesta la formación de una identidad política, no solo en cuanto al tipo de gobierno, sino también a la formación de la sociedad" (2013: 271), y destaca la necesidad de tener en cuenta el papel de estos discursos en la formación de los nuevos Estados nacionales de la América independiente. 
En el marco del proyecto político del Chile independiente, la educación sería un asunto público fundamental, especialmente en el momento de auge del republicanismo:

Con la Independencia, la educación no es «una» política, sino que «es» política porque se le concibe desde y en función de la nueva soberanía. [...] La soberanía popular requería de ciudadanos virtuosos, de la virtud cívica de la república clásica y, porque era popular, requería de un pueblo formado en esa virtud (Serrano, 2010:30).

Bajo esta premisa, primero con Domingo F. Sarmiento y especialmente desde los años 40 del XIX, se multiplicaron los esfuerzos tendientes a difundir y mejorar la educación chilena. Entre otros hitos, bajo la dirección de Manuel Montt, primero como ministro de Instrucción Pública y luego como presidente (1851-1861), se incrementó el número de escuelas y se instauró la gratuidad de la educación primaria, mientras que José Victorino Lastarria, Francisco Bilbao y otros, en época liberal (1841-1876), promovieron la masificación popular de la educación a través de la Sociedad de la Igualdad.

Si la educación era un asunto de interés público, la enseñanza de la lengua espańola, idioma materno de la élite chilena y mayoritario del resto de la población, respondía a la necesidad de dotar a los ciudadanos de una de las herramientas centrales para desenvolverse en una sociedad unificada y centralizada (Mayorga, 2010). Las gramáticas, ortografías y diccionarios del Chile decimonónico, en este sentido, pueden ser interpretados como "escrituras disciplinarias", como hace Velleman (2013). Lo que seńala Narvaja de Arnoux a propósito de las gramáticas escolares del Cono Sur, bien vale para el caso que nos ocupa:

Linguistic unification through a standardized language and the spread of lettered culture were necessary prerequisites to accomplish those social, political and economic goals. The production of school grammars in the first half of the nineteenth century, which went hand in hand with the initial expansion of the educational system, helped meet precisely those goals. They were part of a process [...] that introduced the teaching of Spanish grammar in elementary school [...] and supported the imposition of a standard variety as the common language of the nation (Narvaja de Arnoux, 2013:152).

Entre los primeros debates lingüístico-ideológicos que se dieron en suelo chileno, los relativos a la ortografía (Contreras, 1993) tenían relación precisamente con la necesidad de alfabetizar a la población para asegurar su capacidad de participar en el sistema educativo. La reforma ortográfica propuesta por Andrés Bello y Juan García del Río en 1823 responde manifiestamente a una necesidad educativa del contexto poscolonial (Velleman, 2013). Andrés Bello, figura central de la conformación 
lingüístico-ideológica del XIX chileno, puso la enseñanza de la lengua en un lugar central del proyecto político chileno, por su instrumentalidad en la consecución del orden que anhelaba fervientemente. Como señalan Jaksic, Lolas y Matus (2013: 7-8), Bello consideraba necesaria la alfabetización para que los ciudadanos pudieran ejercer su derecho a sufragio, así como para que pudieran comprender y observar la ley. Las ideas de Domingo F. Sarmiento iban en una dirección similar, aunque, partían de supuestos distintos. Sarmiento, como buen romántico, deseaba la masificación de la educación, lo cual se encontraba estrechamente asociado a su concepción de la lengua como un patrimonio popular; la imperfección que percibía en el sistema ortográfico vigente impedía, en su parecer, dicho ideal (Torrejón, 1989). En su análisis de los métodos de enseñanza de lectura usados en Chile, de 1842, concluyó que "la enseńanza eficaz de la lectura era obstaculizada por un sistema ortográfico que no reflejaba correctamente la pronunciación de los estudiantes" (Velleman, 2004: 45). En consecuencia, debía reformarse la ortografía vigente, aunque sus argumentos no eran puramente didácticos, sino que también incluían un fuerte componente de independentismo cultural, que a su vez se fundamentaba en su pensamiento lingüístico de inspiración herderiana.

El énfasis educativo de la actividad metalingüística de la primera mitad del siglo XIX chileno se prolonga hacia las décadas posteriores. Ya entrada la segunda parte del siglo, aparecen en Valparaíso las Correcciones lexigráficas sobre la lengua castellana de Valentín Gormaz (1860), que llevan por subtítulo dedicadas a la instrucción primaria y que responde al propósito de "procurar un aprendizaje práctico sin el engorro de estudios dilatados" (Gormaz, 1860: vi). Hacia el último cuarto del siglo, se publica el primer diccionario de provincialismos chileno, el Diccionario de chilenismos de Zorobabel Rodríguez (1979 [1875]). A esta obra seguirían varias de índole similar. El propósito didáctico es manifiesto en el Diccionario de chilenismos: la "incorrección con que en Chile se habla y escribe la lengua espańola" se explica por el "gran vacío que hay en la enseñanza de la Gramática castellana” (Rodríguez, 1979 [1875]: vii), vacío a cuyo remedio se propone contribuir el autor con la obra en cuestión.

\section{SAAVEDRA Y VALDERRAMA: LENGUAJE Y EDUCACIÓN}

\section{José Ramón Saavedra}

José Ramón Saavedra, a la fecha de publicación de sus trabajos sobre lenguaje, ejercía como presbítero, dedicado al catecismo y el estudio de la teología. El hecho de que un religioso se dedicara a los estudios del lenguaje no era poco común en el Chile de la época (piénsese en los sacerdotes Camilo Ortúzar y Manuel Antonio Román, autores de sendas obras de prescripción idiomática), ya 
que se trataba de un estamento que participaba activamente de las discusiones intelectuales y que, previo a la Independencia, tenía a su cargo la instrucción y con ello su planificación y reflexión.

En el caso particular de Saavedra, sus trabajos lingüísticos tienen origen en una reacción a la obra gramatical de Andrés Bello: su Gramática elemental de la lengua española (1859) pretendía reemplazar a la publicada en 1845 por el chileno-venezolano. Saavedra planteó su Gramática como un texto específicamente destinado a la enseńanza escolar (acusando de paso un "extravío" de la gramática bellista respecto de dicho objetivo). En 1859, Saavedra presentó ante la Facultad de Filosofía y Humanidades de la Universidad de Chile su Gramática, para que dicha institución apoyara el reemplazo de la obra de Bello por la propia en el contexto escolar, propuesta rechazada por el Consejo de forma rotunda y con fuertes críticas de Eduardo Vargas Fontecilla, quien en los años siguientes sería rival del autor. Tras varios intentos fallidos de que su texto fuera aceptado, publica un último intento de defender sus posturas idiomáticas: su Censura de la Gramática de la Lengua Castellana del señor don Andrés Bello (1863), en la que se esfuerza por deslegitimar el valor pedagógico del texto de Bello y, de paso, saca a flote sus pugnas con Vargas Fontecilla. Sin embargo, cabe destacar que el mismo Saavedra estaba plenamente consciente de la importancia e influencia del chileno-venezolano, aunque considera que su éxito se ha debido a su prestigio personal más que a la calidad de su propuesta lingüístico-educativa.

Ya entrando en las ideas lingüísticas de Saavedra, podemos encontrar sus influencias mencionadas explícitamente en una cita al pie de página de su Gramática. Menciona con frecuencia a pensadores como Juan José Arbolí, Balmes, Novoa, Hermosilla, Avendaño y, muy especialmente, al religioso Isidoro Fernández Monje (véase Eilers, 2010, para un panorama general de los gramáticos españoles del siglo XIX). Este último se había dedicado a la enseñanza y había creado un corpus no menor de textos gramaticales, que tuvieron uso en España (principalmente su Curso elemental de la lengua española, de 1854), y que fue utilizado por Saavedra para la elaboración de su Gramática, a tal punto que Rojas Carrasco (1940), en su recuento moderno de la lingüística chilena, la considera un retroceso pues es solo una reelaboración de la obra de Fernández Monje.

La selección de autores que realiza Saavedra para erigirlos como base de su doctrina gramatical es reveladora de un aspecto importante de su idea de la lengua: se trata principalmente de estudiosos espańoles ligados a la teología, situados en una perspectiva castellanizante del español. De hecho, pueden apreciarse atisbos de antibellismo en Fernández Monje al referirse este a la laxitud con la que el gramático chileno-venezolano proponía reformas que podían devenir en cambios culturales y filosóficos nocivos. Al seguir a Fernández Monje en este punto, Saavedra se ubica en el sector más bien conservador del espectro lingüístico-ideológico. 
Es necesario destacar, por otra parte, el fuerte vínculo que Saavedra ve entre lengua, filosofía y pensamiento humano, idea que hereda a su vez de los autores que cita en su gramática, principalmente de los Arbolí y Balmes. Toma además del primero el molde interpretativo de la gramática general. En este marco de interpretación, el lenguaje es concebido como fiel representación del pensamiento, de manera que mantener estándares de corrección lingüística se equipara a velar por una higiene mental beneficiosa para los ciudadanos y el Estado.

Una vez establecida la importancia de la corrección en el lenguaje, se abre el camino al problema de la autoridad idiomática. Ya mencionamos que el modelo usado por Saavedra pone como centro jerárquico a Espańa, a lo cual se suma la atribución de una autoridad incuestionable y absoluta de la Real Academia Espańola. Pero las cosas no son tan claras cuando hablamos del estudio de la lengua en el siglo XIX chileno, en términos de que la tensión entre autoridad idiomática y estándar deviene en una suerte de inestabilidad en la emisión de ciertos juicios. En el caso de Saavedra, pese a su respeto acérrimo a la normatividad idiomática castellanizante de la Real Academia Espańola, sus ideas sobrepasan su autoridad en determinados casos. Por otro lado, muestra apoyo irrestricto a la institución en términos de ortografía y ortología, declarándose enemigo acérrimo de los llamados "vicios" del español de Chile, tales como el seseo y el yeísmo.

El asunto pasa porque Saavedra sostiene, como sus referentes ideológicos, la idea de que la esencia del estándar lingüístico, más que la Real Academia Española, radica en las ideas tradicionales de la filosofía. La RAE, luego, es respetada por ellos por tender a ser precursora de esas ideas y no tanto por su autoridad, hasta cierto punto, inherente. En definitiva, para el presbítero Saavedra, toda norma está dada por España: sus referentes bibliográficos son todos españoles, su autoridad máxima concreta es una institución española, sus ideales lingüísticos están posicionados en la norma española. En ello se origina además parte de su animadversión hacia Bello: percibe que este último se distancia voluntariamente de la Academia, a pesar del reconocimiento que la institución española había hecho de su Gramática. No cabe duda alguna de que la figura de Bello eclipsó con facilidad a la de Saavedra. En tiempos en que el progreso era lo más importante en términos ideológicos en el continente americano, las ideas de Saavedra supusieron un estancamiento tradicionalista, por lo cual estas habrían de ser ignoradas en muchos sentidos.

Es indudable que, a partir de lo que hemos mencionado, el concepto de lengua que maneja Saavedra está íntimamente vinculado con el horizonte educacional. Esto se puede corroborar con facilidad a partir del hecho de que su Gramática se plantea la ardua tarea de reemplazar a la de Bello como texto de enseñanza, que para él es, pese a su fama, insuficiente como texto escolar. En registros bibliográficos aparecen indexadas su Gramática elemental y su Censura a Bello como las únicas obras de corte lingüístico que se le atribuyen: el resto de sus 
escritos posteriores tuvieron todos que ver con referencias a temáticas religiosas, a excepción de un texto en que reflexionaba sobre la Inquisición como institución, que de todas formas no tenía que ver ya con la lengua como las obras que aquí analizamos. No obstante, su marginación respecto a la lengua no tuvo un correlato necesario con su relación con la enseñanza, ya que se sabe que durante gran parte de su vida ejerció la enseñanza de la doctrina cristiana en los colegios.

Ahora bien, el interés de Saavedra respecto a la educación en relación con una visión propia de la lengua queda de manifiesto particularmente en dos fuentes directas: 1) las citas aclaratorias presentes en su Gramática (más que en su Gramática misma, en la que se aboca a describir fenómenos lingüísticos más que a otra cosa) y 2) su Censura a la Gramática de Bello (que sirve, además, como autodefensa ante el juicio categórico de Vargas Fontecilla).

Dentro de la amplia gama de citas aclaratorias presentes en su texto (un poco más de cincuenta), la mayor parte tiene que ver con dos puntos fundamentales: justificar su doctrina en contraste con las ideas de Bello y señalar el origen ideológico de sus planteamientos. Sea como sea, en ambos tipos de citas Saavedra tiene como objetivo central dar cuenta de la responsabilidad ideológica asociada a la labor que realiza. En este sentido, una de las ideas que más se esmera en sostener es la explicación de por qué su Gramática es mejor que la de Andrés Bello para el uso en las escuelas. De ahí que su preocupación tenga, en buena parte de sus explicaciones, una raigambre didáctica. Esto se aprecia, por ejemplo, en la sección de los modos personales del verbo, donde hace observaciones pedagógicas. Señala nuestro autor que "conviene sobre manera que el alumno se fije en la terminación de las personas de cada tiempo" (Saavedra, 1859: 67), demostrando el interés de que su Gramática llegue a manos de los alumnos de las escuelas. Pero es en una cita de reprimenda a Bello en la que se justifica pedagógicamente su postura lingüístico-educativa:

A pesar del séquito que esta nueva clasificación ha tenido en nuestros colejios, yo hallo más clara la antigua, por las razones siguientes: $1^{a}$ porqué la clasificación del señor Bello tiene que entrar en ciertas afinidades demasiado tenues i casi imperceptibles, que se escapan de la intelijencia de los alumnos. $2^{\text {a }}$ porqué aumentando las clases de verbos regulares [...] se aumentan también las complicaciones i dificultades (Saavedra, 1859: 73).

De aquí que la postura antibellista de Saavedra ya no es sólo lingüística, sino que también guarda relación con la utilidad pedagógica del texto de Bello. Para Saavedra, Bello en muchas oportunidades dificulta, con su terminología y clasificaciones, el aprendizaje de las nociones lingüísticas que deben manejar los estudiantes. A la luz de los hechos, podríamos señalar que el presbítero se opone a la obra de Bello tanto en la dimensión lingüístico-ideológica como en la dimensión pedagógica. 
Tras el segundo rechazo de su Gramática por la Universidad de Chile, Saavedra escribe en 1863 su último y más punzante texto relacionado, la Censura, donde también manifestará su antibellismo. Aquí Saavedra critica a Bello en un punto que tendría resonancia en el futuro (véase, por ejemplo, Rojas Carrasco, 1940): la necesidad en que se veían los profesores de usar textos de apoyo cuando usaban el texto de Bello para impartir clases (sobre la proliferación de textos explicativos de la Gramática de Bello, véase Becco, 1989).

En la Censura de Saavedra, la manifestación de sus ideas pedagógicas se explicita vivazmente en las críticas mordaces a Bello. Señala Saavedra que la Gramática del chileno-venezolano no ha mejorado concretamente la enseñanza como lo plantean sus seguidores, pues las mejoras se deben a asuntos colaterales: por ejemplo, que desde que se estudiaba con la Gramática de Bello, se había aumentado a tres los años de estudio de la lengua (según Saavedra, es natural que entre más ańos se enseñara la lengua, más sabrían los alumnos de ella). Asimismo, señala Saavedra que el progreso del estudio del español es inherente y que preexistía, en términos de avances, a la Gramática de Bello.

Resiente Saavedra que la obra de Bello sea estimada como texto obligatorio de enseñanza por cuestiones de "veneración supersticiosa" y por su aparente "lógica profunda y sensata”. En resumen, el interés principal de Saavedra recae en que se haga un estudio serio del lenguaje desde la infancia, a partir de la enseñanza del buen hablar y de la buena escritura, la cual no halla correlato con los postulados bellistas que, como ya habíamos mencionado anteriormente, son para el presbítero un "extravío de la enseñanza”.

\section{Adolfo Valderrama}

Adolfo Valderrama, un estudioso multifacético como muchos otros de la época (médico cirujano, político, y profesor de retórica), no tiene obras propiamente lingüísticas de su autoría (es decir, no escribió gramáticas o tratados de ortografía), sino más bien comenta los trabajos de otros y la actitud de las autoridades chilenas hacia estos últimos.

Valderrama considera urgente un actuar sobre la educación chilena y lo explicita en su discurso de incorporación a la Facultad de Filosofía y Humanidades (1878) titulado "Necesidad de estudiar la lengua castellana". Desde el título mismo podemos notar que la preocupación principal no se encuentra en el estado de la educación chilena como conjunto, sino más bien en la educación chilena puesta en relación con la lengua española:

Hay preocupaciones entre nosotros que perjudican grandemente el estudio de la lengua castellana: por un lado la falta de hábito de pronunciar bien ciertas letras, como la b y la v, la s y la z (Valderrama, 1878: 28). 
Desde un principio, Valderrama ataca la situación de la enseñanza de la lengua en Chile desde el problema de la norma desobedecida:

¿Hasta qué punto somos culpables nosotros mismos de que este estado de cosas se perpetúe? No nos equivoquemos, señores, no nos hagamos cumplimientos: aceptemos la parte de responsabilidad que nos toca en este asunto para volver sobre nuestros pasos y marchar resueltamente al bien. Examinemos los textos de enseńanza de nuestros colegios y tengamos el valor de poner en el campo del microscopio la leche que damos á nuestra juventud (Valderrama, 1878: 31).

Pero, ¿cuál es este desastroso estado de cosas que nos presenta con tanto resentimiento Adolfo Valderrama? ¿Qué responsabilidades hay en juego? ¿Qué ideal de lengua se está persiguiendo? El autor denuncia en primer lugar una falta en la "falsa idea de que tenemos el estado intelectual de España" (Valderrama, 1878: 28). A partir de esta idea, según Valderrama, se origina una actitud negativa, tanto en el trato del pueblo como del intelectual chileno, hacia la lengua española, que les lleva a considerar indigno su estudio serio, a dejarla merced al antojo de intelectuales que pretenden modificarla de manera que, a juicio del autor, no son apropiadas. Dicho relajo en cuanto a la toma de responsabilidad acerca de la lengua materna provocaría un dańo importante a la identidad y funcionamiento de una lengua ya configurada por los grandes pensadores del Siglo de Oro espańol y custodiada por la Real Academia Española.

La concepción de lengua del autor es muy apegada a una visión generalizada durante el siglo XIX, inspirada en el modelo naturalista de Schleicher y otros filólogos comparatistas: la lengua como un organismo vivo, sujeta a un ciclo natural de nacimiento, desarrollo, decaimiento y eventual muerte (Bynon, 2001). La decadencia y desaparición de las lenguas se deberían específicamente a no realizar los cambios necesarios que mantengan vigente su uso. Valderrama se preocupa por dejar claro que no niega la necesidad del progreso, tanto en el caso de las lenguas como en el de todo asunto humano. Las lenguas estarían sujetas a las mismas dinámicas de progreso que todos los otros elementos de la intelectualidad humana. Sin embargo desde un inicio notamos un conservadurismo lingüístico muy marcado en el autor: junto con expresar esta idea del progreso de las lenguas, llama la atención sobre que progreso no quiere decir degeneración. Su preocupación es que la lengua "no pierda su grandeza en medio de todos los cambios que pueda sufrir" (Valderrama, 1878: 31) y que mantenga su identidad esencial.

Con ello, el autor delimita el espacio de cambio aceptable para la lengua y circunscribe asimismo el círculo de los posibles agentes capacitados para participar en los debates acerca del idioma. Dicho círculo se encuentra reservado para la autoridad castellana que él reconoce: la Real Academia Espańola. En cuanto a la 
pregunta de cómo estudiar la lengua española, su respuesta apunta en dirección similar: bajo una combinación de la lectura de los clásicos españoles y un análisis filosófico del lenguaje. Según el parecer de Valderrama, la orientación de la Academia Espańola, como institución con trayectoria, y el sustento de los clásicos es lo que ha tendido a ser ignorado en Chile con motivo de su reciente Independencia. Valderrama incluso ve, con horror, que el abandono de los clásicos españoles ha tenido como correlato la acogida de modelos franceses.

Valderrama defiende la existencia de una autoridad en materia de lenguaje. Su ausencia, en sus palabras, acarrearía los inconvenientes de una lengua entregada a la anarquía y al desgobierno, una lengua en que cada escritor usaría la lengua según sus caprichos. Lo anterior significaría la ruptura de la unidad de la lengua junto con el desprecio de sus tradiciones y de la rica herencia que esta ha significado a los chilenos. Con ello, Valderrama aventura una concepción del idioma como un cuerpo que contiene una historia (Valderrama, 1986 [1884]: 387). A este respecto, el autor considera a los gramáticos como codificadores de lo ya antes fijado por los grandes escritores, ejemplos del buen uso de la lengua. Menciona como ejemplo de ello el primer diccionario publicado por la Real Academia Espańola (el Diccionario de autoridades, de 1726-1739), en donde se manifiesta la "voz de la autoridad citada" (Valderrama, 1986 [1884]: 389). Esta concepción elitista y literaria de la norma se contrapone a la idea, defendida por románticos como Sarmiento, de que el vulgo pueda ser un aporte en materia de lengua. Dicha idea ya había sido presentada en sus trabajos sobre historia de la poesía chilena, en relación con la poesía popular:

Las más ligera ojeada sobre la poesía popular demuestra el poco caso que nuestro pueblo hace a la lengua; pero al mismo tiempo es fácil reconocer en su poesía una espontaneidad y una gracia singulares. Se ve siempre la falta de instrucción, completo desconocimiento del arte (Valderrama, 1912: 210).

Valderrama hace su ingreso tanto a la Facultad de Filosofía y Humanidades de la Universidad de Chile como al Consejo de Instrucción Pública en un periodo en que ya habían circulado varias propuestas de reforma ortográfica, las más importantes la propuesta de Bello y García del Río en el año 1827 y la de Sarmiento instaurada en Chile el año 1844. El autor destaca varias similitudes entre ambas, pero podemos notar su actitud negativa hacia las reformas bellistas. Al momento de producción del texto que analizamos, se desarrolla la discusión acerca de si se seguía utilizando la ortografía de Bello o si, en palabras de Valderrama, se reconocía el error y se daba por fin la preferencia al sistema ortográfico de la Real Academia Española. Valderrama intenta quitarle a la Academia su imagen de institución anquilosante al atribuirle más bien precaución. Bello, según nuestro autor, habría estado de acuerdo con la actitud prudente de la Academia ante doctrinas nuevas 
como la que él presenta. En ello se ve un reconocimiento por parte de Valderrama a la figura de autoridad que significa Bello en Chile.

\section{CONCLUSIONES}

Los autores estudiados, intelectuales representativos de los participantes en las discusiones lingüísticas del Chile decimonónico, se sitúan en el área conservadora del espectro lingüístico-ideológico del contexto posindependentista. En este sentido, ambos sostienen una actitud bastante reacia a las numerosas propuestas reformistas de otros intelectuales, que consideran nocivas para el proceso educativo de la nación chilena. Asimismo, ambos autores tienen una idea de estándar lingüístico inclinado hacia el referente peninsular, encarnado en la autoridad de la Real Academia Española y los clásicos literarios españoles. Es importante destacar, en lo relativo a este punto, que Valderrama tiene mayor apego a la Academia que Saavedra. Este último se toma la libertad de crear por su cuenta una gramática (aunque se plantee como texto de enseñanza) mientras que Valderrama se limita a defender un seguimiento fiel e irrestricto de la gramática aprobada por la Real Academia Española y todo documento que cuente con el apoyo de dicha autoridad. En ese sentido podemos ver cómo, pese a su conservadurismo, Saavedra no puede evitar hacer un trabajo propio devenido en la mayoría de los casos de las ideas de otros particulares y no necesariamente desde la Academia como fuente directa.

Si bien las posturas de los autores estudiados se diferencian efectivamente en un nivel práctico, podemos encontrar una matriz común de pensamiento en que la relación entre educación y lenguaje juega un papel central aunque no aislado de otros elementos culturales:

Figura 1. Matriz de pensamiento de los autores en torno a lenguaje y educación

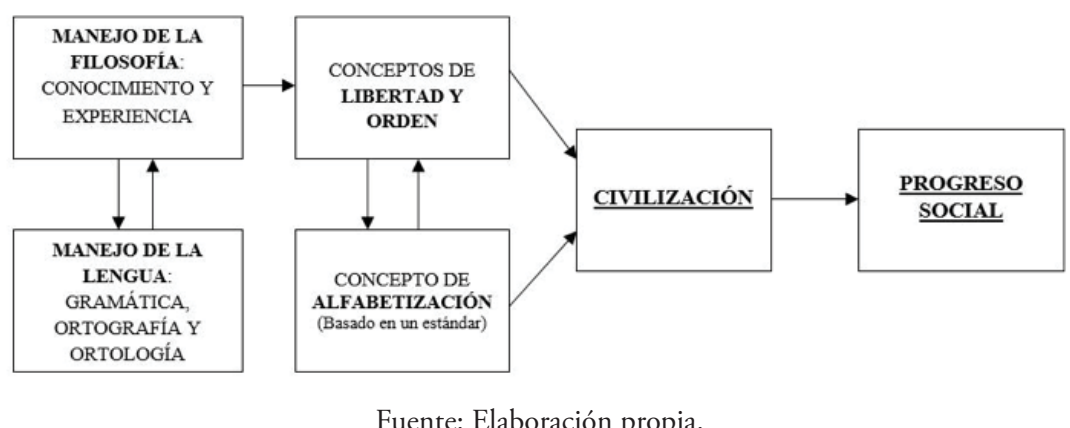


El esquema de la Figura 1 intenta mostrar que el pensamiento lingüístico de estos autores estuvo marcado por una interrelación de conceptos y disciplinas que, al aunarse, devinieron en el desarrollo de sus principales ejes ideológicos en cuanto a necesidades sociales (la libertad y el orden por un lado, y la alfabetización por otro) y cuyo fin máximo era la creación de un orden social civilizado y progresista, alejado de los peligros de la anarquía política. Para ello, desde luego, tenían que establecerse estándares, implementados mediante la educación pública, y eso fue lo que supuso la confrontación de ideales: las problemáticas se suscitaron en temas como dónde situar el estándar lingüístico, qué modelo (europeo) de orden intelectual seguir, cómo y a quiénes educar, etc.

Los autores coinciden en que la lengua es un poderoso instrumento político que cumple un rol ordenador social. En ese sentido, la matriz ideológica central se desvía ampliamente en esta parte del análisis de cada autor: el estándar para llevar a cabo el rol ordenador de la lengua será el punto en conflicto, puesto que a nivel social (y no solo lingüístico) la sensación que había quedado desde la Independencia en relación con España había entrado en una crisis de representatividad.

\section{REFERENCIAS}

Anderson, Benedict. Comunidades imaginadas: Reflexiones sobre el origen y la difusión del nacionalismo. México, D.F.: FCE, 1993.

Becco, Jorge Horacio. Bibliografía de Andrés Bello. Caracas: La Casa de Bello, 1989.

Blommaert, Jan (ed.), Language Ideological Debates. Berlin/New York: Mouton de Gruyter, 1999.

Bynon, Theodora. "The Synthesis of Comparative and Historical Indo-European Studies: August Schleicher". In Sylvain Aroux, E. F. K. Koerner, HansJosef Niederehe y Kees Versteegh (eds.), History of the Language Sciences / Geschichte der Sprachwissenschaft / Histoire des ciences du langage. Volume 2. Berlin / New York: Walter de Gruyter, (2001): 1223-1239.

Cid, Gabriel. "La nación bajo examen. La historiografía sobre el nacionalismo y la identidad nacional en el siglo XIX chileno", Polis 32 (2012): 329-350.

Contreras, Lidia. Historia de las ideas ortográficas en Chile. Santiago de Chile: DIBAM, 1993. 
Eilers, Vera. "La gramática española en el siglo XIX. Gramáticos en diálogo". Aspectos del desarrollo de la lingüistica española a través de los siglos. Vera Eilers, Kirsten Süselbeck y Katharina Wieland (eds.), Hamburg: Helmut Buske, (2010): 31-48.

Gormaz, Valentín. Correcciones lexigráficas sobre la lengua castellana en Chile. Valparaíso: Imprenta del Comercio, 1860.

Huisa, José Carlos. "La impronta política en la primera lexicografía hispanoamericana: republicanismo y antirrepublicanismo", Lexis 37/2 (2013): 269-303.

Jaksic, Iván, Fernando Lolas y Alfredo Matus. "Presentación". Andrés Bello: Gramática de la libertad. Textos sobre lengua y literatura. En Iván Jaksic, Fernando Lolas y Alfredo Matus (comps.), Santiago de Chile: Facultad de Filosofía y Humanidades de la Universidad de Chile, (2013): 7-18.

Mayorga, Rodrigo. "Un nuevo camino de la A a la Z: Enseñanza y aprendizaje de la lectoescritura en la escuela primaria chilena del siglo XIX", Revista Pensamiento Educativo 46-47 (2010): 265-284.

Metzeltin, Miguel. "La construcción discursiva de la República de Chile", Boletín de Filología 46/1 (2011): 239-253.

Narvaja de Arnoux, Elvira. "Grammar and the state in the Southern Cone in the nineteenth century". In José del Valle (ed.), A Political History of Spanish. The Making of a Language. Cambridge: Cambridge University Press, (2013): 152-166.

Rodríguez, Zorobabel. Diccionario de chilenismos. Edición facsimilar. Valparaíso: Editorial Universitaria de Valparaíso, 1979 [1875].

Rojas Carrasco, Guillermo. Filología chilena. Guía bibliográfica y crítica. Santiago de Chile: Imprenta y Literatura Universo, 1940.

Saavedra, José Ramón. Gramática Elemental de la lengua española. 2a edición. Santiago de Chile: Imprenta de la Opinión, 1859.

Saavedra, José Ramón. Censura de la Gramática de la lengua castellana del señor don Andrés Bello. Santiago de Chile: Imprenta de la Opinión, 1863. 
Serrano, Sol. "Educar al nuevo soberano. Chile entre 1810 y 1814", Bordón 62/2 (2010): 29-38.

Swiggers, Pierre. "La historiografía de la lingüística: Apuntes y reflexiones", Revista Argentina de Historiografía Lingüistica 1/1 (2009): 67-76.

Torrejón, Alfredo. "Andrés Bello, Domingo Faustino Sarmiento y el castellano culto de Chile", Thesaurus 44/3 (1989): 534-558.

Valderrama, Adolfo. "Instruccion pública: Elojio de don Justo Florian Lobeck; necesidad de estudiar la lengua castellana: Discurso de incorporacion a la Facultad de Humanidades, el 26 de marzo de 1878", Anales de la Universidad de Chile 53/1 (1878): 242-288.

Valderrama, Adolfo. Obras escogidas/ compilado por Enrique Nercasseau y Morán. Santiago de Chile: Imprenta Barcelona, 1912.

Valderrana, Adolfo. "La cuestión ortográfica". En Alfonso Calderón (ed.), Don Adolfo Valderrama. Santiago de Chile: Academia Chilena de la Lengua, 1986 [1884].

Velleman, Barry L. "Antiacademicismo lingüístico y comunidad hispánica: Sarmiento y Unamuno”. En José del Valle y Luis Gabriel-Stheeman (eds.), La batalla del idioma: La intelectualidad hispánica ante la lengua. Madrid/Frankfurt: Iberoamericana/Vervuert, (2004): 35-65.

Valleman, Barry L. "Bello y las 'escrituras disciplinarias': Diccionarios, gramáticas, ortografías". Ponencia presentada en el I Simposio de la Cátedra Andrés Bello. Universidad de Chile, Chile, 2013. 